\title{
Estimación y alteraciones químicas de suelos tiomórficos con la aplicación de cal en invernadero
}

\author{
Estimation and chemical alterations of tiomórficos soils with \\ lime application in greenhouse \\ Enrique Combatt Caballero ${ }^{*}$, Víctor Hugo Álvarez V. ${ }^{2}$, Júlio César Lima N. ${ }^{2}$
}

\begin{abstract}
RESUMEN
En los suelos tiomórficos o sulfatados ácidos se originan grandes cantidades de ácidos, producto de la oxigenación y drenaje, como consecuencia hay liberación de $\mathrm{Al}^{3+}$ de compuestos insolubles y el $\mathrm{pH}$ desciende a menos que 3,5. El objetivo fue determinar el efecto de la aplicación de cal agrícola en la variación de propiedades químicas en suelos sulfatados ácidos drenados. Seis muestras de suelos fueron colectados en los deltas de los ríos dulces en el estado de Espírito Santo, Brasil y Valle del Río Sinú en Colombia. El trabajo fue desarrollado en invernaderos y laboratorio de fertilidad del Departamento de Suelos de la Universidad de Federal de Viçosa. La cal agrícola utilizada consistió en una mezcla de carbonato de calcio $(\mathrm{CaO}=54 \%)$ y carbonato de magnesio $(\mathrm{MgO}=$ $39,3 \%$ ) en relación molar de 4:1. Para este estudio se utilizaron 14 ecuaciones que presentan diferentes parámetros químicos, como $\mathrm{pH}$, materia orgánica, acidez potencial $(\mathrm{H}+\mathrm{Al})$, saturación de bases $(\mathrm{SB}), \mathrm{Fe}^{2+}, \mathrm{Mn}^{2+}$ y Al ${ }^{3+}$ extraído por varios métodos. Los resultados indican que se necesitan diferentes dosis de cal agrícola, para modificar las características químicas de los diferentes suelos sulfatados ácidos. De acuerdo con las ecuaciones de regresión cuadrática y su utilización para estimar un 40\% de la producción máxima de materia seca es necesario la aplicación de dosis de calcáreo entre 7,03 y $156 \mathrm{t} \mathrm{ha}^{-1}$ para obtener pH 5,0 y reducir el $\mathrm{Al}^{3+}$ intercambiable entre 0,139 y $5,37 \mathrm{cmol}_{\mathrm{c}} \mathrm{dm}^{-3}$. Además al estimar un $80 \%$ de la producción máxima de materia seca es necesario aplicar entre 9,36 y $190 \mathrm{t} \mathrm{ha}^{-1}$ con lo que se lograría alcanzar pH 5,5 y reducir el Al ${ }^{3+}$ intercambiable entre 0 y $1,691 \mathrm{cmol}_{\mathrm{c}} \mathrm{dm}^{-3}$.

Palabras clave: calcáreo, suelos sulfatados ácidos, características químicas, aluminio, ecuaciones.
\end{abstract}

\begin{abstract}
In tiomórficos soils or acid sulphate soils are produced large amounts of acids, product the oxygenation and drainage, as consequence there are release of $\mathrm{Al}^{3+}$ and the $\mathrm{pH}$ drops to less than 3.5. The objective was to determine the effect of the application of agricultural lime in the variation of chemical properties in tiomórficos soils drained. Six soil samples were collected in the deltas of the rivers sweet in the state of Espírito Santo - Brazil and Río Sinú Valley in Colombia. The work was developed in greenhouse and laboratory the Department the soil Federal University of Viçosa. The chemical product that was used consisted of a mixture of calcium carbonate $(\mathrm{CaO}=54 \%)$ and magnesium carbonate $(\mathrm{MgO}=39.3 \%)$ in molar ratio of 4:1. For this study were used 14 equations having different chemical parameters such as $\mathrm{pH}$, organic matter, potential acidity $(H+A l)$, saturation bases $(S B)$, $\mathrm{Fe}^{2+}, \mathrm{Mn}^{2+}$ and $\mathrm{Al}^{3+}$ extracted by several methods. The results indicate that different dosage of agricultural lime are necessary, to modify the chemical characteristics of different tiomórficos soils. According to the quadratic regression equations than were used to estimate $40 \%$ of the maximum dry matter production, is necessary the application of doses of liming between 7.03 and $156 \mathrm{tha}^{-1}$ to obtain $\mathrm{pH} 5.0$ and lower the $\mathrm{Al}^{3+}$ interchangeable between 0.139 and $5.37 \mathrm{cmolc} \mathrm{dm}^{-3}$. In addition for estimated a $80 \%$ of the maximum dry matter production, it is necessary to apply between 9.36 and $190 \mathrm{t} \mathrm{ha} \mathrm{a}^{-1}$, with its which would be achieved to reach $\mathrm{pH} 5.5$ and reduce the $\mathrm{Al} \mathrm{l}^{3+}$ interchangeable between 0 and 1,691 $\mathrm{cmolc}^{-3}$.
\end{abstract}

Key words: limestone, acid sulphate soils, chemical, aluminum, equations.

1 Universidad de Córdoba. Departamento de Ingeniería Agronómica y Desarrollo Rural, Facultad de Ciencias Agrícolas. Montería-Córdoba, Colombia.

2 Departamento de Suelos. Universidade Federal de Viçosa. Viçosa, Minas Gerais, Brasil.

* Autor por correspondencia: ecombatt@correo.unicordoba.edu.co; ecombatt@fca.edu.co

Fecha de Recepción: 27 Octubre, 2016.

Fecha de Aceptación: 24 Octubre, 2017. 


\section{Introducción}

Las alteraciones en las propiedades químicas que ocurren en los suelos sulfatados ácidos actuales, por prácticas de manejo son complejas y poco conocidas por los investigadores y productores agropecuarios, en las regiones interiores de los países de Colombia y Brasil.

La acidez que se origina por la producción de ácido sulfúrico y altas concentraciones de aluminio ha sido registrada como las principales barrera químicas para el crecimiento de las raíces y desarrollo vegetal para alcanzar alta productividad agropecuaria en regiones tropicales con suelos sulfatados ácidos interiores. Dent y Dawson (2000) indican que los suelos tiomórficos (suelos sulfatados ácidos) cuando drenados son extremadamente limitantes al uso agrícola. Esto ocurre porque cuando expuestos al $\mathrm{O}_{2}$ atmosférico $\mathrm{u}$ otros agentes oxidantes hay liberación de $\mathrm{Al}^{3+}, \mathrm{Fe}^{2+}$, $\mathrm{Mn}^{2+}, \mathrm{SO}_{4}^{2}=\mathrm{y} \mathrm{H}^{+}$. Además, en estas condiciones se presentan procesos de oxidación de sulfuros que traen como consecuencia la producción de acidez y baja productividad a los cultivos. Elisa et al. (2011) explican que entre los principales problemas agronómicos asociados a los suelos sulfatados ácidos se encuentra, la toxicidad debido a la presencia de $\mathrm{Al}^{3+}$ y deficiencia de nutrientes.

En las regiones interiores de Colombia y Brasil, suelos sulfatados ácidos potenciales fueron drenados hace aproximadamente cuatro décadas, con el objetivo de ampliar la frontera agrícola, debido a la proximidad de grandes centros urbanos y mayor necesidad de alimentos. Con este cambio de la vocación agrológica, se empezaron a presentar modificaciones en sus propiedades químicas, entre ellas la reacción del suelo $(\mathrm{pH})$ fue la característica química que presentó un descenso, originando $\mathrm{pH}$ menores que 3,5; altas concentraciones de $\mathrm{Al}^{3+}$ con $5 \mathrm{cmol}_{\mathrm{c}} \mathrm{kg}^{-1}$ y sulfatos tan altos como $2.000 \mathrm{mg}$ $\mathrm{kg}^{-1}$, respectivamente. Shamshuddin (2010) expone que cuando el $\mathrm{pH}$ de los suelos es menor que 3,5 se presentan altas concentraciones de $\mathrm{Al}^{3+}$ que son tóxicas para el cultivo del arroz.

El origen de estas condiciones químicas para los productores ha sido poco entendida, porque después de drenado los suelos no presentaban limitante alguna, pero con la ampliación del drenaje, uso continuo de implementos agrícolas y mecanización de suelos, la oxidación del suelo se incrementó y el pH de los suelos se transformó en notablemente ácido y los cultivos presentaron rendimientos muy bajos. Masulili y Utomo (2010) reportaron que los suelos sulfatados ácidos tienen alta cantidad de hierro y sulfatos, presentes principalmente en el mineral pirita y, además, cuando esos suelos son drenados se origina ácido sulfúrico, hierro, aluminio $\mathrm{y}$ otros minerales pesados que son peligrosos para la vida de plantas y organismos.

Con las condiciones de alta acidez y altas concentraciones de $\mathrm{Al}^{3+}, \mathrm{Fe}^{2+}$ y $\mathrm{Mn}^{2+}$ presente en estos suelos, los agricultores comenzaron a utilizar la aplicación de cales agrícolas para disminuir el efecto fitotóxico de estos elementos y aumentar la disponibilidad de algunos nutrientes. Manejo químico, sin conocimiento previo de las modificaciones en las propiedades químicas que se presentarían en los suelos. Según Hammarstrom et al. (2003) cuando se aplica calcita en los suelos se forman precipitados de oxihidróxidos de $\mathrm{Al}^{3+}$ y $\mathrm{Fe}^{2+}$, además se presenta precipitación de los sulfatos en forma del mineral yeso.

La aplicación de cal agrícola a suelos ácidos, tiene la función de neutralizar el $\mathrm{Al}^{3+}$, proporcionar $\mathrm{Ca}^{2+}$ y $\mathrm{Mg}^{2+}$ a las plantas y consecuentemente elevar el $\mathrm{pH}$ y la capacidad productiva del suelo. Rosilawati et al. (2014) bajo condiciones de humedad encontraron que el pH aumentó de 3,43 a 4,02 debido a la aplicación de $6 \mathrm{tha}^{-1}$ de caliza al suelo. Ernani et al. (1998) indican que la disponibilidad de nutrientes se traduce en aumento de rendimiento de los cultivos, un requisito básico para viabilizar económicamente la demanda actual de productos alimenticios y mejorar la sustentabilidad de la actividad agrícola. Ward et al. (2002) afirmaron que entre las técnicas de manejo comúnmente empleadas para la neutralización de la acidez de los suelos sulfatados ácidos potenciales actuales, se encuentran los materiales alcalinos como la cal. Shamshuddin et al. (1991) informaron que las estrategias para aumentar la producción en suelos ácidos, incluye la aplicación de carbonato de calcio, porque se reduce la actividad $\mathrm{Al}^{3+}$ por precipitación como gibbsita $\left(\mathrm{Al}(\mathrm{OH})_{3}\right)$, pero la cantidad de cal requerida para neutralizar la acidez es alta y costosa, por tanto poco práctica.

La información que se ha generado mediante investigaciones sobre la caracterización químicas de estos suelos, con prácticas de enmiendas química es bastante escasa y hasta inadecuada en los países como Brasil y Colombia, por lo anterior el objetivo de la investigación fue determinar 
el efecto de la aplicación de cal agrícola en la variación de parámetros químicos de suelos sulfatados ácidos actuales.

\section{Materiales y métodos}

La investigación se realizó en invernaderos y laboratorio de química y fertilidad de suelos del Departamento de Suelos de la Universidade Federal de Viçosa en Brasil.

Las muestras de suelos fueron colectadas en la región del delta del Río Dulce, en el que fueron seleccionados varios perfiles de suelos enumerados como P2, P3, P4 y P5 en la hacienda Agril en Aracruz y el perfil P1 fue colectado en la región del Valle del Suruaca, Linhares en el estado de Espírito Santo, Brasil. Además fueron recogidas muestras de suelos de otro perfil (P6) en el valle del Río Sinú en Córdoba, Colombia. En cuanto a la clasificación taxonómica de los perfiles, esta fue realizada utilizando la clasificación propuesta por la Embrapa (2006) como Organosolos y Gleissolo tiomórficos típicos y Sulfic Endoaquepts por la SSS (2006).

Para la investigación se utilizaron siete muestras de suelos colectadas de 0-20 y 20-40 cm de profundidad. En el estado de Espírito Santo, Brasil fueron recogidas seis muestras de $0-20 \mathrm{~cm}$ en los perfiles P2, P3, P4 y P5 y de 20-40 cm en el perfil P1. Asimismo, fue en el Valle del Río Sinú en Córdoba, Colombia fue colectada una muestra de 20-40 cm el perfil P6. Las muestras de suelos fueron secadas al ambiente, desterronadas, molidas y posteriormente pasadas por un tamiz con malla de $2 \mathrm{~mm}$ (TFSA) para sus análisis químicos en laboratorio.

\section{Métodos de análisis de los suelos}

En la fracción fina $(<2 \mathrm{~mm})$ de cada suelo fueron realizados los siguientes análisis: fracciones granulométricas y conductividad eléctrica, según métodos descritos por EMBRAPA (1999), $\mathrm{pH}$ cuantificado en agua $\left(\mathrm{pH} \mathrm{H}_{2} \mathrm{O}\right)$ y en $\mathrm{KCl} 1 \mathrm{~mol} \mathrm{~L}^{-1}$ $(\mathrm{pH} \mathrm{KCl})$ relación suelos: solución de 1:2,5. El contenido de carbono de compuestos orgánicos (CO) fue analizado vía combustión seca en analizador elementar (CHNS/O), modelo Perkin Elmer, PE2400. La acidez potencial $(\mathrm{H}+\mathrm{Al})$ fue evaluada según método descrito por Defelipo e Ribeiro (1991). La capacidad de intercambio catiónico (CTC) fue determinada por el método $\mathrm{NH}_{4} \mathrm{OAc} 1 \mathrm{~mol} \mathrm{~L}^{-1}$ a pH 7 y el S disponible, fue extraído con $\mathrm{Ca}\left(\mathrm{H}_{2} \mathrm{PO}_{4}\right)_{2}$, $500 \mathrm{mg} \mathrm{L}^{-1}$ de $\mathrm{P}$, en HOAc $2 \mathrm{~mol} \mathrm{~L}^{-1}$ y determinado por turbidimetría (Hoeft et al., 1973) (Tabla 1).

Para evaluar el $\mathrm{Al}^{3+}$ intercambiable fueron utilizados los siguientes extractores: $\mathrm{KCl} 1 \mathrm{~mol} \mathrm{~L}^{-1}$ (Buurman et al., 1996), $\mathrm{CuCl}_{2} 0,5 \mathrm{~mol} \mathrm{~L}^{-1} \mathrm{pH} 2,8$ (Jou y Kamprat, 1979) y LaCl $0,33 \mathrm{~mol} \mathrm{~L}^{-1} \mathrm{pH} 4,0$ (Hargrove y Thomas, 1981), además del Al fueron cuantificados los contenidos de Fe y Mn.

Para la evaluación de los contenidos de $\mathrm{Al}^{3+}$, $\mathrm{Fe}^{2+}$ y $\mathrm{Mn}^{2+}$ extraíbles fueron colocados $5 \mathrm{~cm}^{3} \mathrm{de}$ suelos y $75 \mathrm{~mL}$ de los extractores en Erlenmeyer de

Tabla 1. Caracterización química de los suelos tiomórficos utilizados en el estudio.

\begin{tabular}{|c|c|c|c|c|c|c|c|c|c|c|}
\hline $\operatorname{Perfil}^{(1)}$ & $\begin{array}{c}\text { Muestras } \\
\text { de suelo }\end{array}$ & Textura $^{(2)}$ & $\mathrm{CE}$ & $\mathrm{pH}_{(\mathrm{H} 2 \mathrm{O})}$ & $\mathrm{pH}_{(\mathrm{KCl})}$ & $\mathrm{H}+\mathrm{Al}$ & CTC & $\mathrm{CO}$ & S & $\mathrm{Na}$ \\
\hline & \multicolumn{5}{|c|}{$\mathrm{dS} \mathrm{m}^{-1}$} & \multicolumn{2}{|c|}{$\mathrm{cmol}_{\mathrm{c}} \mathrm{dm}^{-3}$} & dag $\mathrm{kg}^{-1}$ & \multicolumn{2}{|c|}{$\mathrm{mg} \mathrm{dm}^{-3}$} \\
\hline $\mathrm{P}_{1}$ & $\mathrm{P}_{1} \mathrm{H} 1$ & fs & 8,36 & 3,0 & 3,0 & 144,8 & 160,9 & 6,75 & 14.803 & 197,3 \\
\hline \multirow[t]{2}{*}{$\mathrm{P}_{2}$} & $\mathrm{P}_{2} \mathrm{H} 1$ & faa & 2,74 & 3,0 & 3,0 & 37,0 & 38,45 & 7,68 & 3.811 & 13,9 \\
\hline & $\mathrm{P}_{2} \mathrm{H} 2$ & fs & 5,57 & 3,0 & 3,0 & 99,7 & 102,8 & 31,90 & 5.574 & 403,8 \\
\hline $\mathrm{P}_{3}$ & $\mathrm{P}_{3} \mathrm{Cg}$ & ar & 5,88 & 3,1 & 3,0 & 7,4 & 7,67 & 1,09 & 650 & 70,6 \\
\hline $\mathrm{P}_{4}$ & $\mathrm{P}_{4} \mathrm{H} 1$ & faa & 1,85 & 3,0 & 3,0 & 33,7 & 34,63 & 13,39 & 2.178 & 12,7 \\
\hline $\mathrm{P}_{5}$ & $\mathrm{P}_{5} \mathrm{Ap}$ & fs & 4,38 & 3,0 & 3,0 & 58,0 & 72,22 & 5,42 & 5.849 & 118,2 \\
\hline $\mathrm{P}_{6}$ & $\mathrm{P}_{6} \mathrm{Bgy}$ & $\mathrm{ma}$ & 2,86 & 3,5 & 3,2 & 29,6 & 56,94 & 6,84 & 1.289 & 94,9 \\
\hline
\end{tabular}

1/ $\mathrm{P}_{1} \mathrm{H} 1$. Horizonte $\mathrm{H} 1$, suelo Organossolo Tiomórfico fíbrico salino. $\mathrm{P}_{2} \mathrm{H} 1$ y $\mathrm{P}_{2} \mathrm{H} 2$. horizontes $\mathrm{H} 1$ y $\mathrm{H} 2$, suelos Organossolo Tiomórfico fíbrico salino. $\mathrm{P}_{3} \mathrm{Cg}$. Horizonte $\mathrm{Cg}$, Gleissolo Tiomórfico Órtico (Salino). $\mathrm{P}_{4} \mathrm{H} 1$. Horizonte H1, suelo Gleissolo Tiomórfico Húmico típico. P Ap. Perfil 5, horizontes A, suelo Gleissolo Tiomórfico húmico sódico. P6Bgy. Horizonte Bgy, suelo Gleissolo Tiomórfico Húmico típico (Sulfic Endoaquepts). ${ }^{2 /}$ Clase textural. faa - franco - arcillo - arenoso; fs - franco - limosa; ar - arena; a - arcilla; ma - muy arcillosa sa; as - arcilla limosa. faa - Franco - Arcillo - Arenoso; fs - Franco - limosa; ar - Arena; a - Arcilla; ma - Muy arcilloso; as - Arcilla limosa. 
$125 \mathrm{~mL}$. La suspensión fue agitada por $10 \mathrm{~min}$ y después dejada en reposo por $16 \mathrm{~h}$, y el sobrenadante fue transferido y filtrado para otro Erlenmeyer de $125 \mathrm{~mL}$, para posterior análisis en ICP-OES.

\section{Tratamientos}

Los tratamientos fueron construidos por mediante de la combinación factorial entre siete suelos y siete dosis de cal agrícola, que fueron distribuidos en un diseño experimental de bloques al azar, con tres repeticiones

Para escoger las dosis de cal agrícola, fueron utilizados los resultados previos de los análisis químicos de caracterización de los suelos (Tabla 1), donde se calculó la necesidad de cal agrícola de acuerdo con varias ecuaciones o criterios (Tabla 2). En seguida, dependiendo de los resultados obtenidos en estas ecuaciones fueron escogidas siete dosis de enmienda agrícola $\left(\mathrm{t} \mathrm{ha}^{-1}\right)$ para cada suelo, incluyendo un testigo $\left(0\right.$ tha $\left.^{-1}\right)$ (Tabla 2). El correctivo utilizado consistió de la mezcla de carbonato de calcio $(\mathrm{CaO}=54 \%)$ y carbonato de magnesio $(\mathrm{MgO}=39,3 \%)$ comerciales, en la relación de 4:1.

En esta etapa del experimento las dosis de cal fueron adicionadas a $2,5 \mathrm{dm}^{3}$ de cada suelo, que fueron encubados en bolsas plásticas por $45 \mathrm{~d}$. Durante este período los suelos fueron mantenidos con humedad próxima a la capacidad de campo, por medio de adiciones de agua destilada.

Después del período de incubación, fueron colectadas submuestras de $0,3 \mathrm{dm}^{3}$ de cada unidad experimental, posteriormente secas al aire, desterronadas y pasadas por tamiz con malla de $2 \mathrm{~mm}$. En estas muestras fueron determinados el $\mathrm{pH}$ en agua $(1: 2,5)$; la acidez potencial $(\mathrm{H}+\mathrm{Al})$

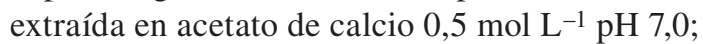
los contenidos de $\mathrm{ca}^{2+}$ y $\mathrm{Mg}^{2+}$ extraídos con $\mathrm{KCl}$ $1 \mathrm{~mol} \mathrm{~L}^{-1}$; el S disponible por el método propuesto por Hoeft et al. (1973) y el P remanescente de acuerdo con Álvarez V. y Fonseca (1990).

Los efectos de las dosis de cal agrícola fueron evaluados por medio de análisis de variancia y modelos de regresión. Los modelos de regresión ajustados tuvieron coeficientes analizados usando el cuadrado medio del error de los análisis de variancia.

\section{Resultados y Discusión}

Las condiciones iniciales del suelo indican que de acuerdo al $\mathrm{pH}$, es notablementemente ácido,
Tabla 2. Ecuaciones para determinar la necesidad de cal agrícola en base a las determinaciones químicas de los suelos y dosis de cal agrícola $\left(\mathrm{t} \mathrm{ha}^{-1}\right)$ obtenidos por ecuaciones y adicionadas en los suelos sulfatados ácidos.

\begin{tabular}{|c|c|c|c|c|c|c|c|}
\hline Métodos & \multicolumn{7}{|c|}{ Ecuaciones o criterios } \\
\hline $\mathrm{M}_{1}$ & \multicolumn{7}{|c|}{$\mathrm{NC}=-1,43+0,9237 *(\mathrm{MO}(6-\mathrm{pH}))$} \\
\hline $\mathrm{M}_{2}$ & \multicolumn{7}{|c|}{$\mathrm{NC}=1,87^{*}(\mathrm{MO}(6-\mathrm{pH}))^{0.731118}$} \\
\hline $\mathrm{M}_{3}$ & \multicolumn{7}{|c|}{$\mathrm{NC}=-0,022+2,7425^{*}((\mathrm{H}+\mathrm{Al})(45-\mathrm{V}) / 100)$} \\
\hline $\mathrm{M}_{4}$ & \multicolumn{7}{|c|}{$\mathrm{NC}=2,906 *((\mathrm{H}+\mathrm{Al})(45-\mathrm{V}) / 100)^{0.80006}$} \\
\hline $\mathrm{M}_{5}^{1 /}$ & \multicolumn{7}{|c|}{$\mathrm{NC}=1,5^{*} \mathrm{Al}_{(\mathrm{KCl})}$} \\
\hline $\mathrm{M}_{6}^{1 /}$ & \multicolumn{7}{|c|}{$\mathrm{NC}=0,08+1,22 * \mathrm{Al}_{(\mathrm{KCl})}$} \\
\hline $\mathrm{M}_{7}$ & \multicolumn{7}{|c|}{$\mathrm{NC}=1,6 *(6,0-\mathrm{pH}) * \mathrm{MOS}$} \\
\hline $\mathrm{M}_{8}{ }^{1 /}$ & \multicolumn{7}{|c|}{$\mathrm{NC}=2 * \mathrm{Al}_{(\mathrm{KCl})}$} \\
\hline $\mathrm{M}_{9}$ & \multicolumn{7}{|c|}{$\mathrm{NC}=0,6 \mathrm{~T}-\mathrm{SB}$} \\
\hline${ }^{2 /} \mathbf{M}_{10}$ & \multicolumn{7}{|c|}{$\mathrm{NC}=2 * \mathrm{Al}_{(\mathrm{Cu})}$} \\
\hline $\mathrm{M}_{11}^{3 /}$ & \multicolumn{7}{|c|}{$\mathrm{NC}=2 * \mathrm{Al}_{(\mathrm{La})}$} \\
\hline $\mathrm{M}_{12}{ }^{4 /}$ & \multicolumn{7}{|c|}{$\mathrm{NC}=2 * \mathrm{SCHA}_{(\mathrm{KCl})}$} \\
\hline $\mathrm{M}_{13}{ }^{5 /}$ & \multicolumn{7}{|c|}{$\mathrm{NC}=2 * \mathrm{SCHA}_{(\mathrm{CuCl} 2)}$} \\
\hline $\mathrm{M}_{14}^{6 /}$ & \multicolumn{7}{|c|}{$\mathrm{NC}=2 * \mathrm{SCHA}_{(\mathrm{LaCl} 3)}$} \\
\hline Suelos & \multicolumn{7}{|c|}{ Tratamientos $\left(\mathrm{t} \mathrm{ha}^{-1}\right)$} \\
\hline P1H1 & 0 & 18,00 & 36,0 & 72 & 144 & 252,0 & 360 \\
\hline $\mathrm{P} 2 \mathrm{H} 1$ & 0 & 5,50 & 11,0 & 22 & 44 & 77,0 & 110 \\
\hline $\mathrm{P} 2 \mathrm{H} 2$ & 0 & 13,75 & 27,5 & 55 & 110 & 192,5 & 275 \\
\hline $\mathrm{P} 3 \mathrm{Cg}$ & 0 & 1.00 & 2,0 & 4 & 8 & 14,0 & 20 \\
\hline P4H1 & 0 & 5,25 & 10,5 & 21 & 42 & 73,5 & 105 \\
\hline P5Ap & 0 & 4,75 & 9,5 & 19 & 38 & 66,5 & 95 \\
\hline P6Bgy & 0 & 3,00 & 6,0 & 12 & 24 & 42,0 & 60 \\
\hline
\end{tabular}

M1, M2, M3 y M4. Modelos lineares de capacidad predictiva de NC para solos ácidos, propuestos por Álvarez, V. (1996). M5 Cate (1965). M6 Catani y Alonso (1969). M7 Defilipo et al. (1972). M8 Kampraht (1967). M9 Sousa et al. (1989). 1/ $\mathrm{Al}$ extraído con $\mathrm{KCl} 1 \mathrm{~mol} \mathrm{~L}{ }^{-1}$. ${ }^{2 /} \mathrm{Al}$ extraído con $\mathrm{CuCl}_{2}$ 0,5 mol L- $1 ;{ }^{3 /} \mathrm{Al}$ extraído con $\mathrm{LaCl}_{3} 0,33$ mol-1; ${ }^{4 /}$ Suma de cationes de hidrólisis ácida (SCHA) Al, Fe y Mn extraídos con $\mathrm{KCl} 1 \mathrm{~mol} \mathrm{~L}^{-1}$; ${ }^{5 /} \mathrm{SCHA} \mathrm{Al}$, Fe y Mn extraídos con $\mathrm{CuCl}_{2}$ 0,5 mol L-1; 6/ SCHA Al, Fe y Mn extraídos con $\mathrm{LaCl}_{3}$ 0,33 mol L-1. Los métodos M12, M13 y M14 fueron propuestos por los autores.

la materia orgánica es alta, el azufre excesivo y fósforo medio. El calcio, magnesio y potasio tienen niveles altos y el sodio presentó niveles bajos. La concentración de aluminio fue evaluada como excesiva y todos los microelementos presentaron altas concentraciones.

La condición de $\mathrm{pH}$ muy ácida y la excesiva acidez intercambiable, se explica por los grandes volúmenes de acidez que son generados por la oxidación de la pirita en medios aeróbicos. Appleyard et al. (2004), indican que a mayor cantidad de pirita oxidada, se incrementan los niveles de $\mathrm{H}_{2} \mathrm{SO}_{4}$ en el suelo, promoviendo la desintegración de las arcillas silicatadas y 
neoformación de sales y óxidos. Este proceso ocasiona la liberación de $\mathrm{H}^{+}$y eleva la concentración de $\mathrm{Mn}^{2+}, \mathrm{Fe}^{2+}$ y $\mathrm{Al}^{3+}$ en solución, que en altas concentraciones son fitotóxicos para los cultivos (Fanning, 2009).

\section{Alteración de las características químicas de los suelos después de aplicación de calcáreo y la incubación}

\section{Contenido de Ca y Mg intercambiable}

Hubo incremento de los contenidos de los elementos en todos los suelos dependiendo de las dosis de calcáreo adicionadas, variando de 10 a $96,4 \mathrm{cmol}_{\mathrm{c}} \mathrm{dm}^{-3}$ para $\mathrm{Ca}^{2+}$ y de 7,23 a $35,7 \mathrm{cmol}_{\mathrm{c}} \mathrm{dm}^{-3}$ para $\mathrm{Mg}^{2+}$ en los suelos que recibieron las mayores dosis de calcáreo (Tabla 3 ). En estos suelos el P1H1 presenta los atributos típicos de los suelos sulfatados ácidos y necesita mayor cantidad de calcáreo para neutralizar la acidez, seguido por el suelo $\mathrm{P} 2 \mathrm{H} 2$.

En los suelos P2H1 y P3Cg, con altos contenidos de arena y bajo poder tampón, los contenidos fueron encontrados entre 5,3 y $31,1 \mathrm{cmol}_{\mathrm{c}} \mathrm{dm}^{-3}$ para $\mathrm{Ca}^{2+}$ y entre 0,84 y $19,25 \mathrm{cmol}_{\mathrm{c}} \mathrm{dm}^{-3}$ para $\mathrm{Mg}^{2+}$, explicado por las menores dosis de calcáreo aplicadas en estos suelos. Campanharo et al. (2007) encontraron incremento significativo en los contenidos de $\mathrm{Ca}^{2+}$ y $\mathrm{Mg}^{2+}$ intercambiable en suelos en función de la aplicación de calcáreo dolomítico. Según Shamshuddin et al. (1991) la presencia de grandes cantidades de $\mathrm{Ca}^{2+}$ en los suelos reduce el efecto toxico de altos contenidos de $\mathrm{Al}^{3+}$.

Los datos fueron ajustados por los modelos de regresión cuadrática para $\mathrm{Ca}^{2+}$ y raíz cuadrática para $\mathrm{Mg}^{2+}$ (Tabla 3). Con el uso de estas regresiones estimadas y al evaluar la necesidad de calcáreo que se necesita para 40 y $80 \%$ de la producción máxima de materia seca estimada con las ecuaciones de regresión fueron obtenidos los contenidos de $\mathrm{Ca}^{2+} \mathrm{y} \mathrm{Mg}^{2+}$ que se necesitan tener en el suelo para alcanzar esas producciones. En este sentido, para $\mathrm{Ca}^{2+}$ fueron estimados contenidos mayores de $8 \mathrm{cmol}_{\mathrm{c}} \mathrm{dm}^{-3} \mathrm{y} \mathrm{Mg}^{2+}$ mayor de $2 \mathrm{cmol}_{\mathrm{c}} \mathrm{dm}^{-3}$, contenidos que son suficientes para suplir los requerimientos nutricionales de los cultivos que puedan ser establecidos en estas condiciones.

Efecto del calcáreo sobre el pH. Los mayores valores de $\mathrm{pH}$, fueron obtenidos en los suelos donde fueron aplicados las mayores dosis de calcáreo (Tabla 4), con pequeña variabilidad entre suelos tamponados y poco tamponados. Andreotti et al.

Tabla 3. Contenidos de $\mathrm{Ca}^{2+}$ y $\mathrm{Mg}^{2+}$ intercambiable y sus ecuaciones en función de las dosis de calcáreo adicionadas en suelos tiomórficos de Brasil y Colombia.

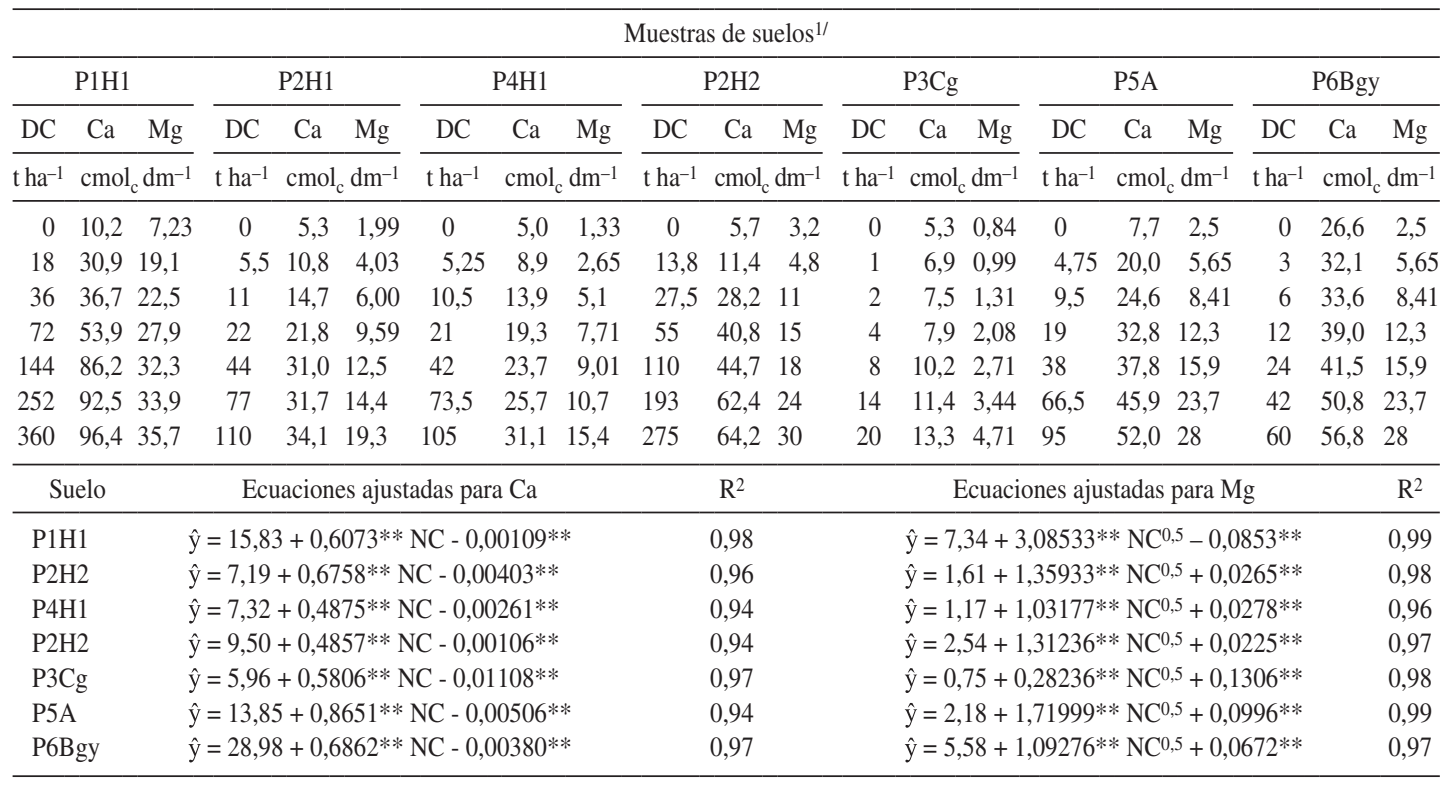

1/ Muestras de suelos colectadas después de 45 días de la aplicación de los tratamientos. ** Significativos a $1 \%$. 
Tabla 4. Valores y ecuaciones de $\mathrm{pH}$ ajustadas en función de las dosis de calcáreo adicionadas en suelos tiomórficos de Brasil y Colombia.

\begin{tabular}{|c|c|c|c|c|c|c|c|c|c|c|c|c|c|}
\hline \multicolumn{14}{|c|}{ Muestras de suelos ${ }^{1 /}$} \\
\hline \multicolumn{2}{|c|}{ P1H1 } & \multicolumn{2}{|c|}{$\mathrm{P} 2 \mathrm{H} 1$} & \multicolumn{2}{|c|}{ P4H1 } & \multicolumn{2}{|c|}{$\mathrm{P} 2 \mathrm{H} 2$} & \multicolumn{2}{|c|}{$\mathrm{P} 3 \mathrm{Cg}$} & \multicolumn{2}{|c|}{ P5A } & \multicolumn{2}{|c|}{ P6Bgy } \\
\hline $\mathrm{DC}$ & $\mathrm{pH}$ & DC & $\mathrm{pH}$ & DC & $\mathrm{pH}$ & $\mathrm{DC}$ & $\mathrm{pH}$ & DC & $\mathrm{pH}$ & $\mathrm{DC}$ & $\mathrm{pH}$ & $\mathrm{DC}$ & $\mathrm{pH}$ \\
\hline $\mathrm{tha}^{-1}$ & $1: 2,5$ & $\mathrm{tha}^{-1}$ & $1: 2,5$ & $\mathrm{t} \mathrm{ha}^{-1}$ & $1: 2,5$ & $\mathrm{tha}^{-1}$ & $1: 2.5$ & $\mathrm{t} \mathrm{ha}^{-1}$ & $1: 2.5$ & $\mathrm{tha}^{-1}$ & $1: 2.5$ & $\mathrm{tha}^{-1}$ & $1: 2.5$ \\
\hline 0 & 2,31 & 0 & 2,56 & 0 & 2,87 & 0 & 2,39 & 0 & 2,86 & 0 & 2,65 & 0 & 3,41 \\
\hline 18 & 2,44 & 5,5 & 2,95 & 5,25 & 3,18 & 13,8 & 2,90 & 1 & 3,08 & 4,75 & 2,71 & 3 & 3,53 \\
\hline 36 & 2,82 & 11 & 3,27 & 10,5 & 3,59 & 27,5 & 3,80 & 2 & 3,38 & 9,5 & 2,86 & 6 & 3,74 \\
\hline 72 & 3,26 & 22 & 4,30 & 21 & 4,85 & 55 & 5,10 & 4 & 3,96 & 19 & 3,12 & 12 & 4,21 \\
\hline 144 & 5,05 & 44 & 5,88 & 42 & 5,71 & 110 & 6,04 & 8 & 5,53 & 38 & 3,92 & 24 & 4,96 \\
\hline 252 & 6,15 & 77 & 6,20 & 73,5 & 6,20 & 193 & 6,33 & 14 & 5,97 & 66,5 & 5,03 & 42 & 5,76 \\
\hline 360 & 6,52 & 110 & 6,43 & 105 & 6,41 & 275 & 6,51 & 20 & 6,20 & 95 & 5,96 & 60 & 5,96 \\
\hline \multirow{2}{*}{ Suelo } & \multirow{2}{*}{\multicolumn{6}{|c|}{ Ecuaciones ajustadas }} & \multicolumn{7}{|c|}{$\mathrm{NC}\left(\mathrm{t} \mathrm{ha}^{-1}\right)$} \\
\hline & & & & & & & $\mathrm{R}^{2}$ & $\mathrm{pH} 5,0^{2 \prime}$ & & $\mathrm{pH} 5,5^{3 /}$ & & $\mathrm{pH}^{4 /}$ & $\mathrm{pH}^{5 /}$ \\
\hline $\mathrm{P} 1 \mathrm{H} 1$ & \multicolumn{6}{|c|}{$\hat{\mathrm{y}}=2,07116+0,023733 * * \mathrm{NC}-0,00003 * * \mathrm{NC}^{2}$} & 0,98 & 153 & \multicolumn{3}{|c|}{190,2} & & \\
\hline $\mathrm{P} 2 \mathrm{H} 2$ & \multicolumn{6}{|c|}{$\hat{\mathrm{y}}=2,49232+0,092488 * * \mathrm{NC}-0,00052 * * \mathrm{NC}^{2}$} & 0,98 & 33,5 & \multicolumn{3}{|c|}{42,8} & 5,16 & 5,62 \\
\hline P4H1 & \multicolumn{6}{|c|}{$\hat{\mathrm{y}}=2,87137+0,087671 * * \mathrm{NC}-0,00050 * * \mathrm{NC}^{2}$} & 0,97 & 29,4 & \multicolumn{3}{|c|}{39,0} & 5,46 & 6,47 \\
\hline $\mathrm{P} 2 \mathrm{H} 2$ & \multicolumn{6}{|c|}{$\hat{\mathrm{y}}=2,59206+0,041886^{* *} \mathrm{NC}-0,00010^{* *} \mathrm{NC}^{2}$} & 0,96 & 68,8 & \multicolumn{3}{|c|}{87,8} & 6,17 & 6,81 \\
\hline $\mathrm{P} 3 \mathrm{Cg}$ & \multicolumn{6}{|c|}{$\hat{\mathrm{y}}=2,72054+0,406251 * * \mathrm{NC}-0,01168 * * \mathrm{NC}^{2}$} & 0,98 & 7,0 & \multicolumn{3}{|c|}{9,3} & 4,07 & 5,64 \\
\hline $\mathrm{P} 5 \mathrm{~A}$ & \multicolumn{6}{|c|}{$\hat{\mathrm{y}}=2,55934+0,034902 * * \mathrm{NC}+0,00001 * \mathrm{NC}^{2}$} & 0,99 & 56,9 & \multicolumn{3}{|c|}{66,5} & 3,18 & 3,66 \\
\hline P6Bgy & \multicolumn{6}{|c|}{$\hat{\mathrm{y}}=3,31535+0,084940 * * \mathrm{NC}-0,00067 * * \mathrm{NC}^{2}$} & 0,99 & 24,6 & \multicolumn{3}{|c|}{35,8} & 3,42 & 4,08 \\
\hline
\end{tabular}

${ }^{1 /}$ Muestras de suelos colectadas después de 45 días de la aplicación de los tratamientos. *, ** Significativos a 5 y $1 \%$. ${ }^{2 /}$ y ${ }^{3 /} \mathrm{NC}$ estimada para alcanzar $\mathrm{pH}$ de 5 y 5,5. ${ }^{4 /}$ y ${ }^{5 /} \mathrm{pH}$ estimado con 40 y $80 \%$ de la NC para obtener la máxima producción de materia seca.

(2001) exponen que los efectos benéficos de la aplicación de cal agrícola en el desarrollo de los cultivos son debidos a la elevación del $\mathrm{pH}$ del suelo y reducción del $\mathrm{Al}^{3+}$ intercambiable, elevación de los contenidos de $\mathrm{Ca}^{2+}$ y $\mathrm{Mg}^{2+}$, y aumento de la disponibilidad de $\mathrm{P}$ para las plantas.

En los suelos $\mathrm{P} 1 \mathrm{H} 1$ y $\mathrm{P} 2 \mathrm{H} 2$ fueron, necesarias entre 275 y 360 t ha $^{-1}$ de calcáreo para aumentar el $\mathrm{pH}$ de 2,3 a valores próximos de 6,5. Esta cantidad excesiva o muy elevada conlleva a la situación donde los altos contenidos de $\mathrm{Ca}^{2+} \mathrm{y} \mathrm{Mg}^{2+}$ resultantes de la disolución del calcáreo ocasionan un incremento de la actividad de estos cationes, dificultando la absorción del $\mathrm{K}^{+}$, principalmente en el inicio del crecimiento del cultivo. Entre los efectos negativos del sobreencalamiento se presenta principalmente la precipitación de varios nutrientes del suelo, como fósforo, zinc, cobre, manganeso y hierro, siendo recomendada sus aplicaciones en la forma foliar.

El modelo de regresión cuadrático fue el modelo matemático que mejor se ajustó con el incremento del pH debido a la aplicación de calcáreo en los suelos tiomórficos. De acuerdo con las ecuaciones de regresión (Tabla 4), se estimaron dosis de calcáreo entre 7,03 y $156 \mathrm{t} \mathrm{ha}^{-1}$ para obtener $\mathrm{pH}$ 5,0 y entre 9,36 y $190 \mathrm{t} \mathrm{ha}^{-1}$ para alcanzar $\mathrm{pH} 5,5$. Los suelos que necesitan mayor cantidades de calcáreo para conseguir $\mathrm{pH}$ de 5 y 5,5 son $\mathrm{P} 1 \mathrm{H} 1$ y $\mathrm{P} 2 \mathrm{H} 2$ los que presentaron contenidos elevados de $\mathrm{Al}^{3+}$, carbono orgánico y, por tanto, mayor acidez potencial. Suelos, con valores de $\mathrm{pH}$ similares pueden tener diferentes fuentes de acidez ligada a las fases orgánicas e inorgánicas, siendo necesario la aplicación de diferentes dosis de calcáreo para neutralizar la acidez; o sea, el poder tampón de acidez es muy variable en los suelos tiomórficos, por sus características químicas. Shazana et al. (2011) exponen que el aumento del $\mathrm{pH}$ es el resultado de la aplicación de caliza de magnesio al suelo y se debe a la producción de iones hidróxilo que se hidrolizan del calcáreo aplicado.

Con el uso de las regresiones ajustadas para la producción de materia seca en función de la necesidad de calcáreo fueron estimadas dosis entre 1,26 y $85,4 \mathrm{t} \mathrm{ha}^{-1}$ para obtener $40 \%$ de la producción máxima estimada mediante los modelos matemáticos. Con esas cantidades el pH del suelo presentó valores entre 3,18 a 6,17. Para $80 \%$ de 
la producción máxima estimada, las dosis fueron entre 9,78 y $187,5 \mathrm{t} \mathrm{ha}^{-1}$, consiguiendo valores de $\mathrm{pH}$ entre 3,66 y 6,8 .

Disponibilidad de azufre. El contenido de $\mathrm{S}$ disponible disminuyó como consecuencia del aumento de las dosis de calcáreo adicionadas, siendo esta tendencia relacionadas con las características químicas de los suelos. En los suelos sin adición de calcáreo los contenidos oscilaron entre 15,765 y $0,529 \mathrm{~g} \mathrm{dm}^{-3}$ y entre 1,441 y $0,471 \mathrm{~g} \mathrm{dm}^{-3}$ en los suelos con mayores tratamientos de dosis de calcáreo (Tabla 5).

Esta disminución puede estar directamente relacionada con la precipitación de diferentes compuestos de sulfatos, siendo el yeso $\left(\mathrm{CaSO}_{4} \mathrm{H}_{2} \mathrm{O}\right)$, que es el principal mineral que se puede formar en suelos tiomórficos. En estas condiciones con la aplicación de calcáreo se incrementan los contenidos de $\mathrm{Ca}^{2+}$, que al formar un par iónico con el $\mathrm{SO}_{4}=$ precipita el sulfato como $\mathrm{CaSO}_{4}$. Banata et al. (2006) indican que en suelos con altos contenidos de $\mathrm{S}$ y diferentes contenidos de $\mathrm{Ca}^{2+}$, puede ocurrir la precipitación de este elemento en la forma de $\mathrm{CaSO}_{4}$.

Según Seth y Crawford (2000) la aplicación de cal agrícola y otros agentes neutralizantes con $\mathrm{Ca}^{2+}$ tienen el potencial para precipitar yeso, y reducir los contenidos de S. Offeddu et al. (2014) encontraron que la precipitación de yeso junto con la disolución de dolomita es más lento que la del carbonato de calcio.

Basados en las ecuaciones ajustadas (Tabla 5), fue determinado que el modelo de regresión que mejor explica los procesos químicos de disminución de $\mathrm{S}$ disponible con la aplicación de calcáreo en los suelos sulfatados ácidos interiores fue: $\hat{y}$ $=(\mathrm{a}+\mathrm{b} * \mathrm{NC})^{-1 / \mathrm{c}}$.

Con los modelos de regresión que explican las tendencias químicas de los parámetros evaluados, además que fueron utilizados para predecir el 40 y $80 \%$ de la producción máxima, y entre ellos las ecuaciones ajustadas de $\mathrm{S}$, se determinó que los contenidos de $\mathrm{S}$ presentan una disminución de entre 26 y $86 \%$ y entre 76 y $98 \%$ para alcanzar

Tabla 5. Contenidos y ecuaciones de $\mathrm{S}$ disponible ajustadas en función de dosis de calcáreo adicionadas en suelos sulfatados ácidos de Brasil y Colombia.

\begin{tabular}{|c|c|c|c|c|c|c|c|c|c|c|c|c|c|}
\hline \multicolumn{14}{|c|}{ Muestras de suelos ${ }^{1 /}$} \\
\hline \multicolumn{2}{|c|}{ P1H1 } & \multicolumn{2}{|c|}{$\mathrm{P} 2 \mathrm{H} 1$} & \multicolumn{2}{|c|}{$\mathrm{P} 4 \mathrm{H} 1$} & \multicolumn{2}{|c|}{$\mathrm{P} 2 \mathrm{H} 2$} & \multicolumn{2}{|c|}{$\mathrm{P} 3 \mathrm{Cg}$} & \multicolumn{2}{|c|}{$\mathrm{P} 5 \mathrm{~A}$} & \multicolumn{2}{|c|}{ P6Bgy } \\
\hline $\mathrm{DC}$ & $\mathrm{S}^{2 /}$ & $\mathrm{DC}$ & $S$ & $\mathrm{DC}$ & $\mathrm{S}$ & $\mathrm{DC}$ & $\mathrm{S}$ & DC & $\mathrm{S}$ & $\mathrm{DC}$ & $\mathrm{S}$ & $\mathrm{DC}$ & $S$ \\
\hline $\mathrm{t} \mathrm{ha}^{-1}$ & $\mathrm{~g} \mathrm{dm}^{-3}$ & $\mathrm{tha}^{-1}$ & $\mathrm{~g} \mathrm{dm}^{-3}$ & $\mathrm{t} \mathrm{ha}^{-1}$ & $\mathrm{~g} \mathrm{dm}^{-3}$ & $\mathrm{t} \mathrm{ha}^{-1}$ & $\mathrm{~g} \mathrm{dm}^{-3}$ & $\mathrm{tha}^{-1}$ & $\mathrm{~g} \mathrm{dm}^{-3}$ & $\mathrm{t} \mathrm{ha}^{-1}$ & $\mathrm{~g} \mathrm{dm}^{-3}$ & $\mathrm{t} \mathrm{ha}^{-1}$ & $\mathrm{~g} \mathrm{dm}^{-3}$ \\
\hline 0 & 15,765 & 0 & 3,534 & 0 & 1,912 & 0 & 5,666 & 0 & 0,529 & 0 & 6,046 & 0 & 1,247 \\
\hline 18 & 13,610 & 5,5 & 2,201 & 5,25 & 1,475 & 13,8 & 3,596 & 1 & 0,504 & 4,75 & 5,945 & 3 & 1,022 \\
\hline 36 & 12,758 & 11 & 1,596 & 10,5 & 1,085 & 27,5 & 1,937 & 2 & 0,539 & 9,5 & 5,286 & 6 & 0,942 \\
\hline 72 & 9,161 & 22 & 1,053 & 21 & 0,855 & 55 & 9,610 & 4 & 0,567 & 19 & 3,813 & 12 & 0,784 \\
\hline 144 & 4,587 & 44 & 0,805 & 42 & 0,598 & 110 & 8,520 & 8 & 0,573 & 38 & 2,960 & 24 & 0,781 \\
\hline 252 & 1,672 & 77 & 0,751 & 73,5 & 0,542 & 193 & 0,712 & 14 & 0,589 & 66,5 & 2,208 & 42 & 0,672 \\
\hline 360 & 1,145 & 110 & 0,728 & 105 & 0,471 & 275 & 0,674 & 20 & 0,607 & 95 & 1,441 & 60 & 0,675 \\
\hline \multirow{2}{*}{\multicolumn{2}{|c|}{ Suelo }} & \multirow{2}{*}{\multicolumn{4}{|c|}{ Ecuaciones ajustadas }} & & & \multicolumn{6}{|c|}{$\mathrm{S}$ disponible $\left(\mathrm{g} \mathrm{dm}^{-3}\right)$} \\
\hline & & & & & & & $\mathrm{R}^{2}$ & \multicolumn{3}{|c|}{ NC 0,4 ŷ máx } & \multicolumn{3}{|c|}{ NC 0,8 ŷ máx } \\
\hline \multirow{2}{*}{\multicolumn{2}{|c|}{$\begin{array}{l}\mathrm{P} 1 \mathrm{H} 1 \\
\mathrm{P} 2 \mathrm{H} 2\end{array}$}} & \multicolumn{5}{|c|}{$\hat{y}=(0,112971+0,00024 * * N C)^{-1 / 0,22465 * *}$} & 0,98 & & - & & \multicolumn{3}{|c|}{-} \\
\hline & & \multicolumn{5}{|c|}{$\hat{y}=(0,004069+0,00021 * * N C)^{-1 / 0,67643 * *}$} & 0,91 & & 0,730 & & \multicolumn{3}{|c|}{0,581} \\
\hline \multicolumn{2}{|l|}{$\mathrm{P} 4 \mathrm{H} 1$} & \multicolumn{5}{|c|}{$\hat{\mathrm{y}}=(0,021486+0,00029 * * \mathrm{NC})^{-1 / 0,52187 * *}$} & 0,92 & & 0,826 & & \multicolumn{3}{|c|}{0,425} \\
\hline \multicolumn{2}{|l|}{$\mathrm{P} 2 \mathrm{H} 2$} & \multicolumn{5}{|c|}{$\hat{\mathrm{y}}=\left(0,135934+0,00115^{* *} \mathrm{NC}\right)^{-1 / 0,23091^{* *}}$} & 0,94 & & 0,533 & & \multicolumn{3}{|c|}{0,091} \\
\hline \multicolumn{2}{|l|}{$\mathrm{P} 3 \mathrm{Cg}$} & \multicolumn{5}{|c|}{$\hat{\mathrm{y}}=(0,130647-0,00031 * * \mathrm{NC})^{-1 / 0,32441 * *}$} & 0,81 & & 0,546 & & \multicolumn{3}{|c|}{0,571} \\
\hline \multirow{2}{*}{\multicolumn{2}{|c|}{$\begin{array}{l}\text { P5A } \\
\text { P6Bgy }\end{array}$}} & \multicolumn{5}{|c|}{$\hat{\mathrm{y}}=\left(0,423189+0,00076^{* *} \mathrm{NC}\right)^{-1 / 0,09862 * *}$} & 0,97 & & 4,443 & & \multicolumn{3}{|c|}{3,518} \\
\hline & & \multicolumn{5}{|c|}{$\hat{\mathrm{y}}=\left(0,109592+0,00036^{* *} \mathrm{NC}\right)^{-1 / 0,31849^{* *}}$} & 0,79 & & 1,021 & & &, 937 & \\
\hline
\end{tabular}

${ }^{1 /}$ Muestras de suelo colectadas después de 45 días de la aplicación de los tratamientos. ${ }^{2 /}$. El S disponible fue extraído con $\mathrm{Ca}\left(\mathrm{H}_{2} \mathrm{PO}_{4}\right)_{2}$, $500 \mathrm{mg} \mathrm{L}^{-1}$ de $\mathrm{P}$, en HOAc $2 \mathrm{~mol} \mathrm{~L}^{-1}$ y expresado en $\mathrm{g} \mathrm{dm}^{-3}$ debido a la magnitud de los datos encontrados. ** Significativos a $1 \%$. S estimado con 40 y $80 \%$ de la NC para obtener la máxima producción de materia seca. 
40 y $80 \%$ de la producción máxima estimada. Sin embargo, en el suelo $\mathrm{P} 3 \mathrm{Cg}$ se presentó un incremento en los contenidos de $\mathrm{S}$ disponible de entre 8 y 13\%, explicado por la posible liberación de $\mathrm{S}$ de los compuestos minerales y orgánicos. En este suelo fueron aplicadas bajas dosis de calcáreo, debido a las características químicas y bajo poder tampón de acidez.

Contenidos de Al intercambiable y de acidez. potencial. Al observar los contenidos del aluminio intercambiable se verificó que este catión fue completamente neutralizado con la aplicación de la cuarta dosis de calcáreo en todos los suelos (Tabla 6). Por otro lado, se resalta que el suelo P1H1 necesita la mayor cantidad de calcáreo para neutralizar $\mathrm{Al}^{3+}$ intercambiable que es el elemento fitotóxico, presente en los suelos, además de la alta capacidad tampón que se presentan en algunos suelos timórficos.

Esta neutralización del $\mathrm{Al}^{3+}$ puede ser explicada por el proceso de solubilización de la calcita, que incrementa el $\mathrm{pH}$ de la solución del suelo y consecuentemente el $\mathrm{Al}^{3+}, \mathrm{Fe}^{2+}$ y $\mathrm{Mn}^{2+}$ precipitan. Kashir y Yanful (2000) exponen que el tratamiento más común para incrementar el pH y neutralizar la acidez del suelo es el uso de calcáreo, con el que se precipitan compuestos amorfos de óxidos e hidróxidos de Fe y Al. Además de la formación de cristales de yeso que se pueden presentar, además la disolución del calcáreo genera reacciones de intercambio de cationes y precipitan el $\mathrm{Al}^{3+}$ a $\mathrm{pH}$ próximos a 7 .

Según Hammarstrom et al. (2003) cuando se aplica calcáreo en los suelos ácidos se forman precipitados de oxihidróxidos de $\mathrm{Al}$ y Fe, además

Tabla 6. Contenidos de $\mathrm{Al}^{3+}$ y $\mathrm{H}+\mathrm{Al}$ y ecuaciones ajustadas en función de las dosis de calcáreo adicionadas en suelo sulfatados ácidos de Brasil y Colombia.

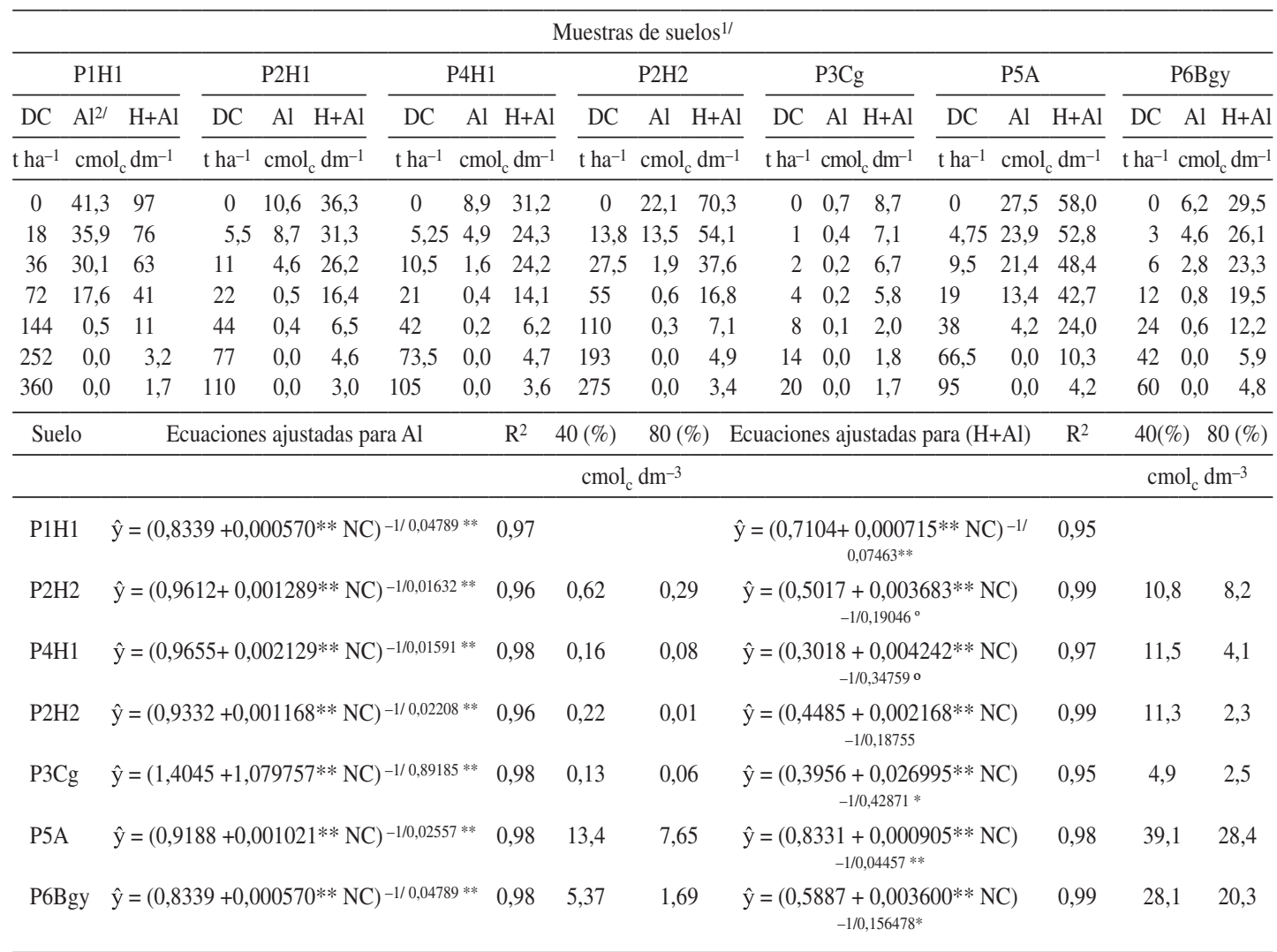

1/Muestras de suelo colectadas después de 45 días de la aplicación de los tratamientos. ${ }^{2 /} \mathrm{Al}$ determinado por espectrofotometría de emisión en IPC-OAS. ${ }^{3 /}$ y ${ }^{4 /}$ Contenidos de Al estimados con 40 y $80 \%$ de la NC para obtener la máxima producción de materia seca. *, **. Significativo a 5 y $1 \%$. 
existe precipitación de los sulfuros en forma del mineral yeso.

El modelo de regresión que explica la disminución de $A l^{3+}$ fue $\hat{y}=\left(a+b^{*} \mathrm{NC}\right)^{-1 / c}$. Con el uso de estas regresiones ajustadas para la determinación de la necesidad de calcáreo se encontró que los contenidos de $\mathrm{Al}^{3+}$ se pueden reducir entre 0,139 y $5,37 \mathrm{cmol}_{\mathrm{c}} \mathrm{dm}^{-3}$ cuando se estima un $40 \%$ y entre 0 y $1,691 \mathrm{cmol}_{\mathrm{c}} \mathrm{dm}^{-3}$ para $80 \%$ de la producción máxima de materia seca estimada, excepto en el suelo $\mathrm{P} 5 \mathrm{~A}$ donde los contenidos de $\mathrm{Al}^{3+}$ se mantienen entre 7,65 y $13,48 \mathrm{cmol}_{\mathrm{c}} \mathrm{dm}^{-3}$. Fageria y Baligar (2005) indican que varios materiales calcáreos pueden ser utilizados para reducir la acidez de los suelos, al contrarrestar los efectos del exceso de iones $\mathrm{H}^{+} \mathrm{y} \mathrm{Al}^{3+}$.

Las dosis de calcáreo aplicadas en los diferentes suelos permiten reducir en más del 95\% la acidez potencial de los suelos $(\mathrm{H}+\mathrm{Al})$, encontrando contenidos entre 1,7 y $3,0 \mathrm{cmol}_{\mathrm{c}} \mathrm{dm}^{-3}$ en los tratamientos con mayores dosis de calcáreo (Tabla 6). Sin embargo, los contenidos de acidez potencial $(\mathrm{H}+\mathrm{Al})$ permanecen más elevados en los suelos $\mathrm{P} 2 \mathrm{H} 2$ y P6Bgy de mayor capacidad tampón. La reducción en la acidez potencial está directamente relacionada con los procesos químicos de precipitación de los principales elementos de hidrolísis ácida presente en los suelos tiomórficos. Según Demchak (2001) cuando se incrementa la alcalinidad en los suelos se presenta mudanza en el $\mathrm{pH}$ y el $\mathrm{SO}_{4}=$ por la precipitación del $\mathrm{Fe}^{2+}$ y $\mathrm{Al}^{3+}$ intercambiable en forma de Jarosite $\left((\mathrm{H}, \mathrm{K}, \mathrm{Na})-\mathrm{Fe}_{3}(\mathrm{OH})_{6}\left(\mathrm{SO}_{4}\right)_{2}\right)$ o Jurbanite $\left(\mathrm{AlOHSO}_{4}\right)$. Totok Suswanto et al. (2007) al aplicar $4 \mathrm{t} \mathrm{ha}^{-1}$ de caliza combinado con fertilizantes orgánicos, encontraron incremento en el rendimiento de arroz, debido a los efectos combinados del aumento de $\mathrm{pH}, \mathrm{Ca}^{2+}$ y $\mathrm{Mg}^{2+}$ intercambiables y la reducción de la concentración de $\mathrm{Al}^{3+}$ y $\mathrm{Fe}^{2+}$ en la solución del suelo.

De acuerdo con lo observado en la Tabla 6, el modelo de regresión que mejor ajuste matemático presentó para la disminución de acidez potencial $(\mathrm{H}+\mathrm{Al})$ cuando aplicadas las dosis de calcáreo en los suelos tiomóficos fue $\hat{y}=(a+b * N C)^{-1 / c}$. Mediante las ecuaciones ajustadas para la determinación de la necesidad de calcáreo, se encontró que los contenidos de $\mathrm{H}+\mathrm{Al}$ se pueden reducir entre $33 \mathrm{y}$ $84 \%$ cuando es estimada una producción del $40 \%$ y entre 51 y $97 \%$ cuando se estima un $80 \%$ de la producción máxima de materia seca.

\section{Conclusiones}

Las dosis de cal agrícola aplicadas en los suelos tiomórficos permiten alcanzar cantidades mayores de 8 y $2 \mathrm{cmol}_{\mathrm{c}} \mathrm{dm}^{-3}$ de calcio y magnesio intercambiables, que son suficientes para el desarrollo de cultivos.

De acuerdo con las ecuaciones de regresión estimadas, se necesitan aplicar dosis de cal agrícola que oscilen entre 7,03 y $156 \mathrm{t} \mathrm{ha}^{-1}$ para obtener un pH 5,0 y entre 9,36 y $190 \mathrm{t} \mathrm{ha}^{-1}$ para alcanzar $\mathrm{pH} 5,5$.

Los modelos o regresiones ajustadas para la determinación de la necesidad de calcáreo, permiten realizar estimaciones mediante las que los contenidos de $\mathrm{Al}^{3+}$ se pueden reducir entre 0,139 y $5,37 \mathrm{cmol}_{\mathrm{c}} \mathrm{dm}^{-3}$ cuando se calcula un $40 \%$ de la producción máxima de materia seca y entre 0 y $1,691 \mathrm{cmol}_{\mathrm{c}} \mathrm{dm}^{-3}$ cuando se estima un $80 \%$ de la producción máxima de materia seca.

Las dosis de calcáreo aplicadas en los diferentes suelos permiten reducir en más del 95\% la acidez potencial $(\mathrm{H}+\mathrm{Al})$ de los suelos tiomórficos.

\section{Agradecimientos}

A la Universidad Federal de Viçosa, al Departamento de Suelos y al Programa de Postgraduación por la oportunidad ofrecida para la realización de la investigación. A la coordinación de perfeccionamiento de la CAPES por el auxilio financiero.

\section{Literatura Citada}

Álvarez V., V.H.; Fonseca, D.M.

1990. Definição de doses de fósforo para a determinação da capacidade máxima de adsorção de fosfato e para ensaios de casa de vegetação. R. Bras. Ci. Solo, 14: 49-55.

Andreotti, M., Souza, E.C.A. de; Crusciol, C. A. C.

2001. Componentes morfológicos e produção de matéria seca de milho em função da aplicação de calcário e zinco. Scientia agrícola, 58 (2): 321-327.
Appleyard, S.; Wong, S.; Willis, J.B.; Angeloni, J.; Watkins, R. 2004. Groundwater acidification caused by urban development in Perth, Western Australia: source, distribution, and implications for management. Australian Journal of Soil Research, 42: 579-585.

Banata, K.M.; Howarib, F.M.; Abdullahc, M.B.

2006. Mineralogy and hydrochemical characteristics of the late marshes and swamps of Hor Al Hammar, Southern Iraq. Journal of Arid Environments, 65: 400-419. 
Buurman, P.; Van, L.B.; Velthor, J.

1996. Manual for Soil and Water Analyses. Backhuys

Publishers. Leiden, The Netherlands. 314 p.

Campanharo, M.; Andrade, M.; Araújo DO Nascimento, C.;

Freire, F.; Tenório, J.

2007. Avaliação de métodos de necessidade de calagem no Brasil. Revista caatinga, 20 (1): 97-105.

Defelipo, B.V.; Ribeiro, A.C.

1991. Análise química do solo. 2. ed. Viçosa, MG, Universidade Federal de Viçosa. 26 p.

Demchak, J.; Morrow, T.; Skousen, J.

2001. Treatment of acid mine drainage by four vertical flow wetlands in Pennsylvania. Geochem Expl Environ Anal, 1 (1): 1-12.

Dent, D.; Dawson, B.

2000. The acid test: an expert system for acid sulphate soils. University of East Anglia. Norwich. 38 p.

Elisa Azura, A.; Shamshuddin, J.; Fauziah, C.I.

2011. Root elongation, root surface area and organic acid exudation by rice seedling under $\mathrm{Al}^{3+}$ and/or $\mathrm{H}^{+}$stress. American Journal of Agricultural and Biological Sciences, 6: 324-331.

\section{EMBRAPA}

1999. Manual de análises químicas de solos, plantas e fertilizantes. Organizador: Fábio Cesar da Silva. Embrapa Comunicação para Transferência de tecnologia. Brasilia, Brasil. 370 p.

Empresa Brasileira de Pesquisa Agropequaria-EMBRAPA 2006. Centro Nacional de Pesquisa de Solos. Sistema Brasileiro de classificação de solos. 2 ed. Río de Janeiro, Embrapa Solos. 306 p.

Ernani, P.; Nascimento, J.; Oliveira, L.

1998. Increase of grain and green matter of corn by liming. Revista Brasileira de Ciência do Solo, 22 (2): 275-280.

Fageria, N.K.; Baligar, V.C.

2005. Enhancing nitrogen use efficiency in crop plants, Adv. Agron, 88: 97-185.

Francesco, G.O.; Jordi, C.; Josep M.S.; Christine, V.P.

2014. Direct nanoscale observations of the coupled dissolution of calcite and dolomite and the precipitation of gypsum. Beilstein J. Nanotechnol, 5: 1245-1253.

Hammarstrom, J.M.; Sibrell, P.L.; Belkin, H.E.

2003. Characterization of limestone reacted with acid-mine drainage in a pulsed limestone bed treatment system at the Friendship Hill National Historical Site, Pennsylvania, USA. Applied Geochemistry, 18: 1705-1721.

Hargrove, W.L.; Thomas, G.W.

1984. Extraction of aluminum from aluminum-organic matter in relation to titratable acidity. Soil Science Society of America Journal, 48: 1458-1460.

Hoeft, R.G.; Walsh, L.M.; Keeney, D.R.

1973. Evaluation of various extractants for available soil sulfur. Soil Sci.Soc. Am. Proc, 37: 401-411.
Juo, A.S.; Kamprath, E.J.

1979. Copper chloride as on extractant for estimating the potentially reactive aluminum pool in acid soils. Soil Science Society of America Journal, 43: 35-58.

Kashir, M.; Yanful, E.K.

2000. Compatibility of slurry wall backfill soils with acid mine drainage. Advances in Environmental Research, 4: 252-268.

Neal, C.; Robson, A.J.; Smith, C.J.

1990. Acid neutralization capacity variations for Hafren forest streams: inferences for hydrological processes. $J$ Hydrol, 121: 85-101.

Masulili, A.; Utomo, W.H.

2010. Rice Husk Biochar for Rice Based Cropping System in Acid Soil 1. The Characteristics of Rice Husk Biochar and Its Influence on the Properties of Acid Sulfate Soils and Rice Growth in West Kalimantan, Indonesia. Journal of Agricultural Science, 2 (1): 39.

Rosilawati, A.K.; Shamshuddin, J. and Fauziah, C.I.

Effects of incubating an acid sulfate soil treated with various liming materials under submerged and moist conditions on $\mathrm{pH}, \mathrm{Al}$ and $\mathrm{Fe}$. African Journal of Agricultural Research, 9 (1): 94-112.

Shamshuddin, J.; Fauziah, C.I.

2010. Alleviating acid soil infertility constraints using basalt, ground magnesium limestone and gypsum in a tropical environment. Malaysian J. Soil Sci, 14: 1-13.

Seth, R.; Crawford, E.

2000. The effects of $\mathrm{pH}$ regulation upon the release of sulfate from ferric precipitates formed in acid mine drainage. Applied Geochemistry, 15: 27-34.

Soil Survey Staff

2006. Keys to soil taxonomy. 9th edition. United States Department of Agricultura (USDA). Natural Resources Conservation Services. 332 p.

Shamshuddin, J.; Che Fauziah, I.; Sharifuddin, H.A.H. 1991. Effects of limestone and gypsum applications to a Malaysian Ultisol on soil solution composition and yields of maize and groundnut. Plant Soil, 134: 45-52.

Shazana, M.; Shamshuddin, J.; Fauziah, C.I.; Syed Omar, S. R. 2011. Alleviating the infertility of an acid sulphate soil by using Ground basalt with or without lime and organic fertilizer Under submerged conditions. Land Degradation \& Development Land Degrad. Develop. Published online, 24(2): 129-140. in Wiley Online Library < wileyonlinelibrary. com>DOI: 10.1002/ldr.1111

Totok, S.; Shamshuddin, J.; Syed Omar, S.R.; Peli Mat and. Teh, C.B.S.

2007. Alleviating an acid sulfate soil cultivated to rice (Oryza sativa) using ground magnesium limestone and organic fertilizer. Jurnal Tanah dan Lingkungan, 9: (1) 1-9.

Ward, N.; Sullivan, L.; Bush, R.

2002. Sulfide oxidation and acidification of acid sulfate soil materials treated with $\mathrm{CaCO}_{3}$ and seawater-neutralised refinery residue. Australian Journal of Soil Research, 40: 1057-1067. 